\title{
ENERGY TRANSFER MECHANISMS IN THE RANQUE-HILSCH VORTEX TUBE
}

\author{
Nolan Dyck \\ Department of Mechanical Engineering \\ The University of Western Ontario \\ London, Ontario, Canada \\ Email:ndyck@uwo.ca
}

\author{
Anthony Straatman \\ Department of Mechanical Engineering \\ The University of Western Ontario \\ London, Ontario, Canada \\ Email: astraatman@eng.uwo.ca
}

\begin{abstract}
The Ranque-Hilsch Vortex Tube (RHVT) is a small tube without any moving parts receiving compressed air from a tangential inlet and exhausting cold air from a small hole at one end of the tube while exhausting hot air from an annular exit at the opposite end. Nearly a century has passed since the RHVT was first patented, and the mechanism responsible for this socalled "temperature separation" remains unclear. The present work tests the hypothesis that kinetic energy is transferred from the stream of air leaving the cold exit to the air stream leaving the hot exit.

To test the hypothesis, a parametric study using an axisymmetric model of the RHVT has been carried out using CFD software, wherein the pressure at the hot exit was varied. The study has been validated against previous experimental and computational models. The results show that the dominant mode of energy transfer between the two streams is work transfer, which supports the hypothesis.
\end{abstract}

Index Terms-Ranque-Hilsch Vortex Tube, Compressible flow, Computational Fluid Dynamics

\section{INTRODUCTION}

Consider the schematic shown in figure 1. A compressible fluid is supplied at the entrance shown at some pressure $p_{\text {in }}$. Provided $p_{\text {in }}$ exceeds $p_{\text {cold }}$ and $p_{\text {hot }}$, some fraction of the inlet mass $\mu_{\mathrm{c}}=\dot{m}_{\text {cold }} / \dot{m}_{\text {in }}$ leaves via the cold exit, while the remaining fraction $1-\mu_{c}$ is exhausted at the hot exit. While this observation is rather unremarkable, many researchers [1], [2], [3], [4] have observed physical experiments in which the static temperature of cold exit stream was significantly lower than that of the inlet stream, while the static temperature of the hot exit stream was significantly higher than that of the inlet stream. This "temperature separation" was first noticed by George J. Ranque [5] in 1922, and is often referred to as the Ranque effect. In a later investigation Hilsch [1] discussed the effects of varying the geometric constraints of the flow boundaries. The vortex tube is now commonly referred to as the Ranque-Hilsch Vortex Tube (RHVT) after the aforementioned researchers. Sometimes the abbreviation is shortened to VT.

While vortex tubes are easy to construct and operate, the mechanism(s) responsible for the temperature separation phenomenon are still unknown, as noted in a recent review by Thakare et al. [6]. Many researchers have conducted studies and proposed theories of operation over the past century, and while much data is available, the complex interaction between fluid mechanics and thermodynamics remains unclear.

Ranque [5] originally suggested that the temperature separation could be explained using the isentropic ideal gas relation, wherein the sudden expansion at the inlet causes a decrease in density and a sharp drop in temperature. Experiments by Gao et al. [7] and Xue et al. [8] indicate this theory significantly over-predicts the temperature drop at the cold exit. Furthermore this does not explain the corresponding temperature increase at the hot exit.

Some numerical studies have revealed the presence of recirculation region(s) at various points within the VT. Both Ahlborn and Groves [2], and Xue and others [9] have suggested multiple re-circulation zones exist, each one behaving as a heat pump to move energy away from the core and towards the periphery. While earlier papers have provided mediocre evidence for this theory, more recent papers reporting the results of steady and unsteady, 3D, RANS and LES results have included streamline plots depicting multiple recirculation zones occurring within short vortex tubes [10], [11], [12], [13]. The re-circulation zones appear to be mobile and sensitive to geometry and boundary conditions.

A third theory states that cold stream transfers kinetic energy to the hot stream through viscous shear. This work transfer theory was first proposed by Hilsch [1], who suggested that 'internal friction' was the mode by which energy is transferred from the axis to the periphery. Aljuwayhel et al. [14] have since analyzed the results of an axisymmetric CFD model and provided quantitative evidence that work transfer from the cold stream to the hot stream is dominant mechanism of energy transfer in the RHVT. More recently, Polihronov et al. [15] and Tlili El May et al. [16] have observed wall shear stress spikes in 3D simulations of the VT, suggesting the cold stream is transferring kinetic energy to the hot stream.

In the present work we aim to quantify the energy transfer between the cold stream and the hot stream, testing our hypothesis that shear work is the primary mechanism of temperature separation. Section II provides the governing equations applicable to the current analyses, section III details the CFD model used, section IV reports the results of our simulations, and section $\mathrm{V}$ analyzes the energy transfer 


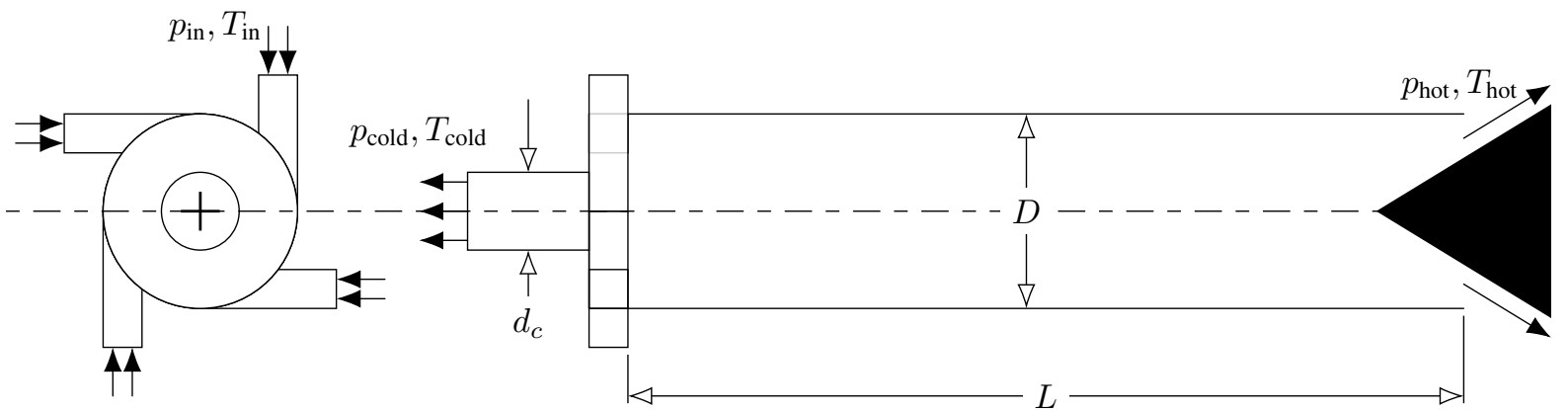

Fig. 1. A schematic of a typical Ranque-Hilsch Vortex Tube.

between the cold and hot streams.

\section{Governing EQUATIONS}

The conservation of mass is

$$
\frac{\partial \rho}{\partial t}+\nabla \cdot(\rho \mathbf{u})=0
$$

where $\mathbf{u}$ is the velocity, $\rho$ is the density, $t$ is time, and $\boldsymbol{\nabla}$ is the gradient operator. The conservation of momentum for a Newtonian fluid where Stokes' hypothesis has been invoked is

$$
\frac{\partial \mathbf{u}}{\partial t}+(\mathbf{u} \cdot \boldsymbol{\nabla}) \mathbf{u}=-\frac{\boldsymbol{\nabla} p}{\rho}+\frac{\mu}{\rho}\left(\nabla^{2} \mathbf{u}+\frac{1}{3} \boldsymbol{\nabla}(\boldsymbol{\nabla} \cdot \mathbf{u})\right),
$$

where $p$ is the thermodynamic pressure and $\mu$ is the dynamic viscosity. The conservation of internal energy is

$$
\rho\left(\frac{\partial \varepsilon}{\partial t}+\mathbf{u} \cdot \boldsymbol{\nabla} \varepsilon\right)=-p(\boldsymbol{\nabla} \cdot \mathbf{u})-\boldsymbol{\nabla} \cdot \mathbf{q},
$$

where $\varepsilon$ is the specific internal energy and $\mathbf{q}$ is the heat flux vector.

\section{A. Auxillary equations}

All fluids analyzed in this work are assumed to obey the ideal gas equation of state:

$$
p=\rho R_{s} T,
$$

where $R_{s}$ is the specific ideal gas constant, and $T$ is the static, absolute temperature.

We will further assume a constant heat capacity for all fluids analyzed in this work, so that the internal energy and enthalpy may be respectively written as

$$
\begin{aligned}
& \varepsilon=c_{v} T, \\
& h=c_{p} T,
\end{aligned}
$$

where $c_{v}$ is the volumetric heat capacity and $c_{p}$ is the isobaric heat capacity. Fluids which obey the ideal gas law and have constant heat capacities are called perfect gases [17].

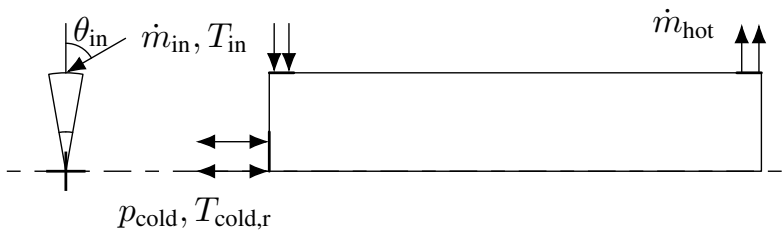

Fig. 2. Simplified VT geometry for CFD study

TABLE I

PARAMETERS OF THE PRESENT CFD STUDY

\begin{tabular}{||l|l||}
\hline Measurement & Value \\
\hline Working tube length [mm] & 106 \\
Working tube inner diameter $[\mathrm{mm}]$ & 11.4 \\
Nozzle total inlet area $\left[\mathrm{mm}^{2}\right]$ & 8.2 \\
Cold exit diameter $[\mathrm{mm}]$ & 6.2 \\
Cold exit area $\left[\mathrm{mm}^{2}\right]$ & 30.3 \\
Hot exit area $\left[\mathrm{mm}^{2}\right]$ & 95 \\
Nozzle angle $\left[{ }^{\circ}\right]$ & 75.48 \\
\hline Inlet conditions & \\
\hline mass flow rate $\left[\mathrm{g} \mathrm{s}^{-1}\right]$ & 8.34 \\
Total Temperature $\left[{ }^{\circ} \mathrm{C}\right]$ & 21.21 \\
\hline
\end{tabular}

\section{CFD STUDY}

To analyze the energy transfer between the hot and cold streams we have performed a CFD analysis of the commercial vortex tube studied by Skye et al. [4]. The simplified vortex tube geometry is visible in Fig. 2, and the relevant geometric parameters are given in Table I.

For each simulation, the mass flow rate at the inlet is $\dot{m}_{\text {in }}=$ $8.34 \mathrm{~g} \mathrm{~s}^{-1}$, the inlet velocity angle is $\theta_{\text {in }}=75.48^{\circ}$, and the inlet total temperature is $T_{\mathrm{in}}=21.21^{\circ} \mathrm{C}$. Skye et al. notes the presence of reverse flow at the cold exit for small cold mass fractions, so an opening boundary condition is applied at the cold exit, where the total temperature of the recirculating air, $T_{\text {cold, },}$, is set to the bulk mean total temperature of the leaving air, $T_{\text {cold. }}$. To replicate the experiment conditions of Skye et al. the cold exit pressure $p_{\text {cold }}$ has been set to the experimentally measured value for each cold mass fraction.

Two non-orthogonal structured grids have been generated 


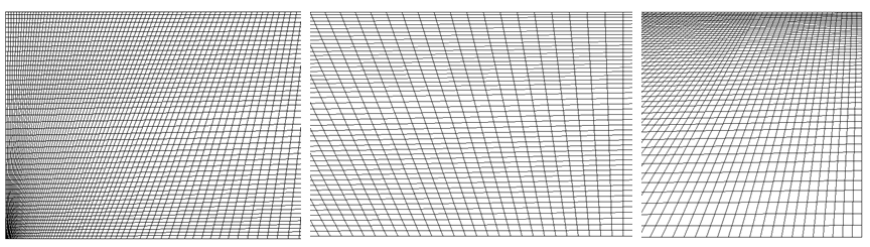

Fig. 3. Sample views of the fine mesh at inlet, middle, and hot outlet end.

using ANSYS Workbench ${ }^{\circledR}$ Meshing Software [18]: a relatively course grid containing 1890 nodes and a fine grid containing 41778 nodes. Sample images of the fine grid are provided in Fig. 3. The $\mu_{\mathrm{c}}=0.208$ case was run using each mesh and the results were compared. The cold exit temperature differed by $0.22 \%$ and the hot exit temperature differed by $0.017 \%$, indicating our results are grid-independent. The fine grid has been used for the remainder of the simulations reported on in this work.

In each simulation convergence was achieved when the rms residuals for the mass, momentum, and turbulent equations were fell below $10^{-6}$, while the energy equation rms residuals fell below $10^{-5}$.

ANSYS-CFX $\odot 16$ [19] has been used to setup and solve each of the simulations. The standard $k-\varepsilon$ turbulence model has been used.

\section{Results AND Discussion}

The measured temperature separation values at the cold and hot exits are defined by

$$
\begin{gathered}
T_{\mathrm{s}, \mathrm{c}}=T_{\text {in }}-T_{\text {cold }} \\
T_{\mathrm{s}, \mathrm{h}}=T_{\text {hot }}-T_{\text {in }}
\end{gathered}
$$

where the total temperatures have been used. The cold and hot temperature separation values have been plotted in Figs. 4 and 5 alongside data obtained from experimental and computational results. The cold exit temperature separation appears to peak at a cold mass fraction of $\mu_{\mathrm{c}} \approx 0.35$, while the hot exit temperature separation steadily increases with cold mass fraction. The 3D, unsteady Large Eddy Simulations conducted by Farouk and Farouk [20] predict the cold stream temperature separation more accurately than the present model.

Despite our less accurate predictions, the present model still closely captures the trend of the experimental data at both the hot and cold exits. A possible explanation for the discrepancy between the present model and Farouk and Farouk's is that the mechanism present in the axisymmetric models is augmented or supplemented by unsteady circumferential variations. In either case, the results of the axisymmetric model can be further analyzed to gain more insight into at least one temperature separation mechanism.

Fig. 6 shows a typical streamline plot of the axisymmetric VT, where several interesting features are present. First, the hot stream occupies only a narrow band around the tube

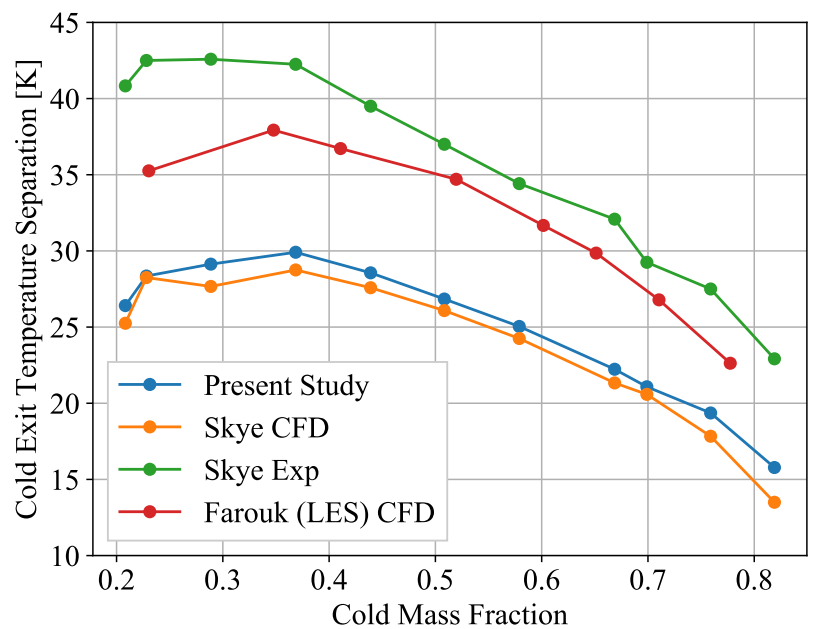

Fig. 4. Cold exit temperature separation.

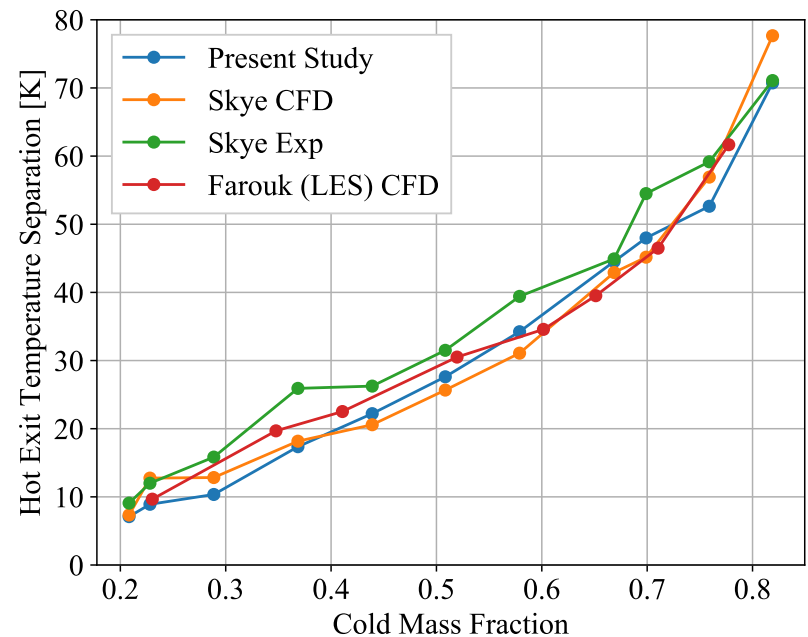

Fig. 5. Hot exit temperature separation.

wall, while the rest of the domain is occupied by the slender reverse flow region of the cold stream. Furthermore there is a recirculation region present in at the cold outlet, caused by the strong radial pressure variation. This recirculation region disappears for cold mass fractions greater than 0.4. Finally, a stagnation streamline connects the inlet to a zero velocity region near the hot outlet. When the stagnation streamline is revolved about the tube axis, it represents a stream surface separating the hot stream from the cold stream.

\section{EnERgy Transfer Modes}

With the converged solution fields at hand, the energy transfer modes across the stagnation streamline may be computed. We follow the same procedure as Aljuwayhel et al. [14], by computing the various energy transfers across the stagnation streamline. The differential energy transfers associated with conduction heat transfer, circumferential shear work, and axial shear work are given by 


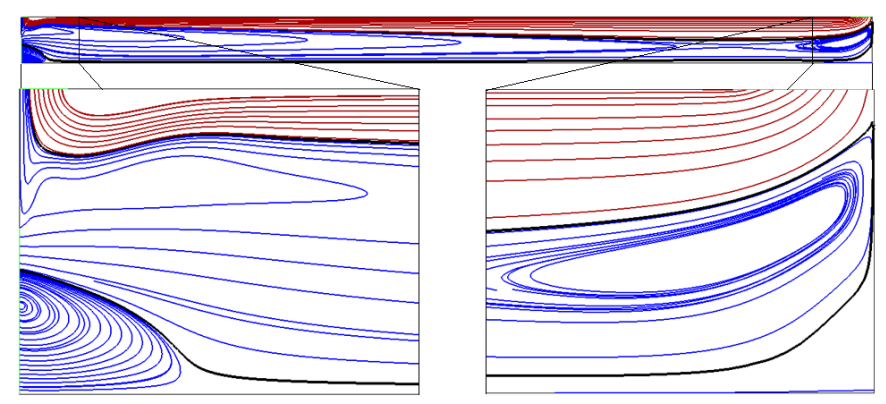

Fig. 6. Streamline plot of axisymmetric CFD results, with a cold mass fraction of 0.208 . Red streamlines proceed from the inlet to the hot exit, blue streamlines terminate at the cold exit, and two stagnation streamlines are represented by thick black lines. The stagnation streamline of interest divides the hot and cold streams.

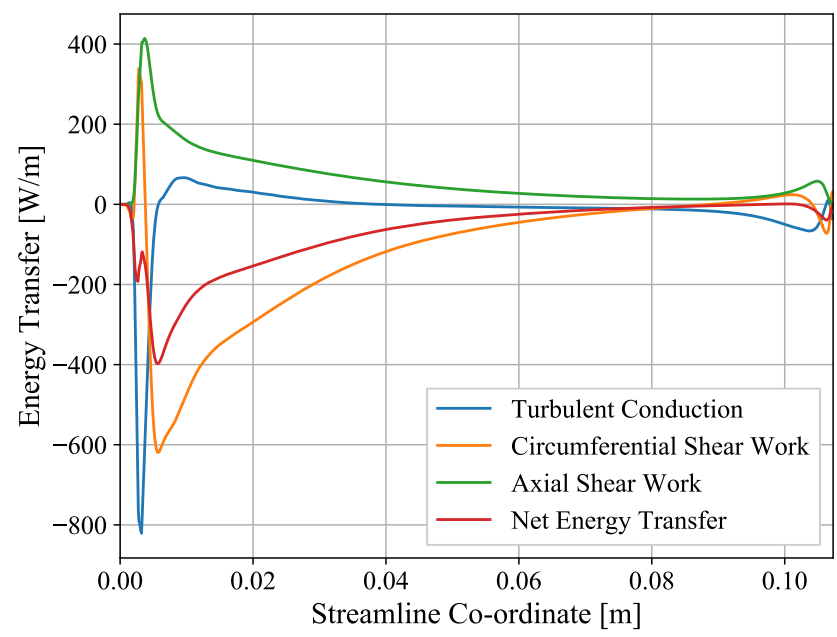

Fig. 7. Energy transfer across the stagnation streamline for the case $\mu_{\mathrm{c}}=$ 0.208

$$
\begin{aligned}
\frac{\partial \dot{Q}}{\partial s} & =-2 \pi k_{\mathrm{eff}} r \frac{\partial T}{\partial n} \\
\frac{\partial \dot{W}_{\theta}}{\partial s} & =-2 \pi \mu_{\mathrm{eff}} r v_{\theta} r \frac{\partial}{\partial n}\left(\frac{v_{\theta}}{r}\right) \\
\frac{\partial \dot{W}_{z}}{\partial s} & =-2 \pi \mu_{\mathrm{eff}} v_{z} r \frac{\partial v_{z}}{\partial n}
\end{aligned}
$$

where $s$ is a streamline co-ordinate, and $n$ is the normal vector pointing towards the hot stream. The contributions from each of these have plotted as a function of the streamline co-ordinate for two cold mass fractions Figs. 7 and 8. In the case of a low cold mass fraction, the majority of the energy transfer between the streams takes place near the inlet, diminishes as the streamsurface contracts towards the axis, and recovers near the hot exit. The circumferential shear work transfer is the dominant energy transfer mechanism. For a cold mass fraction of 0.819 , the majority of the energy transfer takes place near the hot exit.

The total energy transferred via each mechanism can be found by integrating over the stream line, and the results

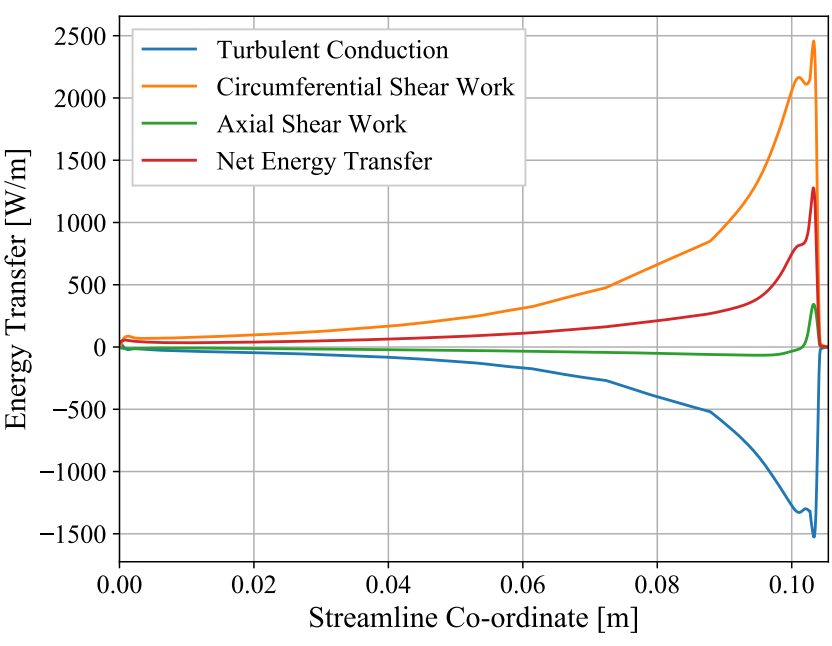

Fig. 8. Energy transfer across the stagnation streamline for the case $\mu_{\mathrm{c}}=$ 0.819

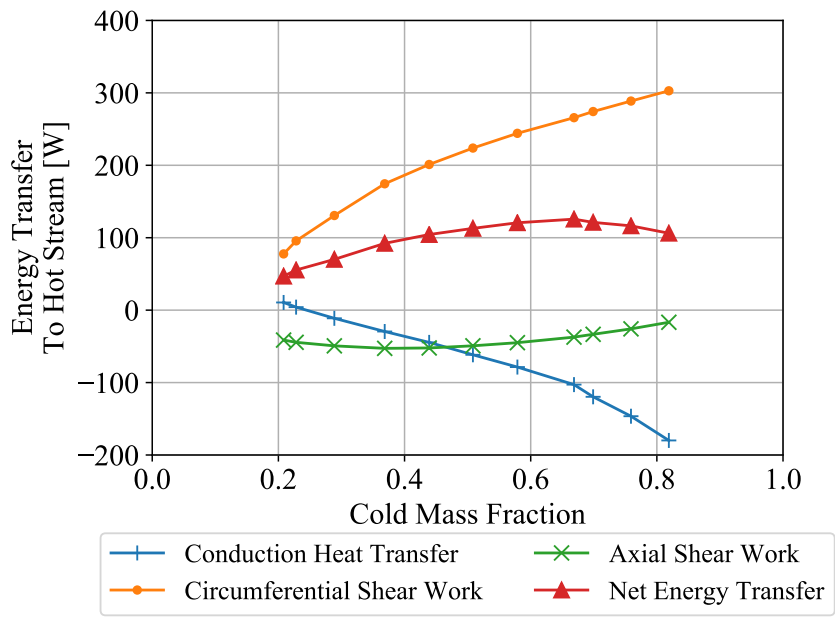

Fig. 9. Total Energy transfer across the stagnation streamlines.

have been plotted as a function of cold mass fraction in Fig. 9. As the cold mass fraction increases, the energy transferred through circumferential shear work and conduction heat transfer increase. Since the energy is transferred in opposite directions, the net effect is only a modest increase in energy transfer.

To validate our findings, we have compared the energy increase of the flow leaving the hot exit with the net energy transfer to the hot stream across the stagnation streamline. The hot stream energy increase has been computed using

$$
\Delta E_{\mathrm{hot}}=\left(1-\mu_{\mathrm{c}}\right) \dot{m} c_{p} T_{\mathrm{s}, \mathrm{h}}
$$

The percent differences between the energy transfer across the stagnation streamline and equation 12 have been plotted in Fig. 10. Overall the discrepancies are modest. It is likely there is a small amount of energy transferred via radial shear at smaller mass fractions since the stagnation streamlines have 


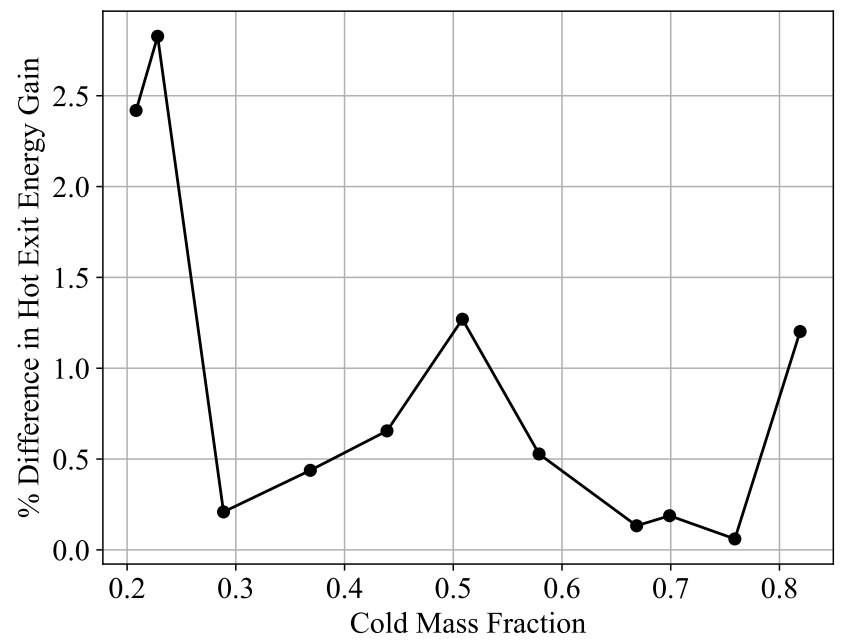

Fig. 10. Percent difference between energy increase computed based on the difference between the inlet and hot exit and the total energy transferred across the stagnation streamline.

the most significant radial variation, which could account for the greater discrepancies at low mass fractions.

\section{CONCLUSION}

In this work we have re-analyzed the vortex tube studied by Skye et al. [4] using an axisymmetric CFD model. It was observed that the trends in temperature separation matched those seen in experiments, though they are less accurate than 3D unsteady CFD models. We have also computed the transfers of kinetic and thermal energies across the stagnation streamline, demonstrating that circumferential shear work transfer is the dominant energy transfer mechanism.

Although the hypothesis has been supported, result is unsatisfying as the study fails to answer the deeper questions about the mechanism behind temperature separation; a complete picture of the energy movement in the system is still elusive, and there aren't any clear indications of which parameters are most important in vortex tube design. Furthermore, recirculation regions near the boundaries are present and cast doubt on the correctness of the velocity field. Future work will focus on better matching the boundary conditions between experiments and computational results.

\section{ACKNOWLEDGMENT}

The authors gratefully acknowledge the financial support from the Natural Sciences and Engineering Research Council of Canada (NSERC).

\section{REFERENCES}

[1] R. Hilsch, "The use of the expansion of gases in a centrifugal field as cooling process," Review of Scientific Instruments, vol. 18, no. 2, pp. 108-113, 1947.

[2] B. Ahlborn and S. Groves, "Secondary flow in a vortex tube," Fluid Dynamics Research, vol. 21, no. i, pp. 73-86, 1997.
[3] K. Dincer, S. Baskaya, B. Z. Uysal, and I. Ucgul, "Experimental investigation of the performance of a Ranque-Hilsch vortex tube with regard to a plug located at the hot outlet," International Journal of Refrigeration, vol. 32, no. 1, pp. 87-94, 2009. [Online]. Available: http://dx.doi.org/10.1016/j.ijrefrig.2008.06.002

[4] H. M. Skye, G. F. Nellis, and S. A. Klein, "Comparison of CFD analysis to empirical data in a commercial vortex tube," International Journal of Refrigeration, vol. 29, no. 1, pp. 71-80, 2006.

[5] G. J. Ranque, "Experiments on expansion in a vortex with simultaneous exhaust of hot air and cold air," J. Phys. Radium, vol. 4, no. 7, pp. 112 $114,1933$.

[6] H. R. Thakare, A. Monde, and A. D. Parekh, "Experimental, computational and optimization studies of temperature separation and flow physics of vortex tube: A review," Renewable and Sustainable Energy Reviews, vol. 52, pp. 1043-1071, 2015. [Online]. Available: http://www.sciencedirect.com/science/article/pii/S1364032115008564

[7] C. M. Gao, K. J. Bosschaart, J. C. H. Zeegers, and A. T. A. M. De Waele, "Experimental study on a simple Ranque-Hilsch vortex tube," Cryogenics, vol. 45, no. 3, pp. 173-183, 2005.

[8] Y. Xue and M. Arjomandi, "The effect of vortex angle on the efficiency of the Ranque-Hilsch vortex tube," Experimental Thermal and Fluid Science, vol. 33, no. 1, pp. 54-57, 2008. [Online]. Available: http://linkinghub.elsevier.com/retrieve/pii/S0894177708000952

[9] Y. Xue, M. Arjomandi, and R. Kelso, "The working principle of a vortex tube," International Journal of Refrigeration, vol. 36, no. 6, pp. 1730-1740, 2013. [Online]. Available: http://dx.doi.org/10.1016/j.ijrefrig.2013.04.016

[10] R. Liew, J. C. H. Zeegers, J. G. M. Kuerten, and W. R. Michalek, "Maxwell's demon in the ranque-hilsch vortex tube," Physical Review Letters, vol. 109, no. 5, pp. 3-6, 2012.

[11] H. R. Thakare and A. D. Parekh, "Computational analysis of energy separation in counter-flow vortex tube," Energy, vol. 85, pp. 62-77, 2015. [Online]. Available: http://dx.doi.org/10.1016/j.energy.2015.03.058

[12] S. E. Rafiee and M. M. Sadeghiazad, "Experimental study and 3D CFD analysis on the optimization of throttle angle for a convergent vortex tube," Journal of Marine Science and Application, vol. 15, no. 4, pp. 388-404, 2016. [Online]. Available: http://link.springer.com/10.1007/s11804-016-1387-1

[13] V. Bianco, A. Khait, A. Noskov, and V. Alekhin, "A comparison of the application of RSM and LES turbulence models in the numerical simulation of thermal and flow patterns in a double-circuit Ranque-Hilsch vortex tube," Applied Thermal Engineering, vol. 106, pp. 1244-1256, 2016. [Online]. Available: http://linkinghub.elsevier.com/retrieve/pii/S1359431116310092

[14] N. F. Aljuwayhel, G. F. Nellis, and S. A. Klein, "Parametric and internal study of the vortex tube using a CFD model," International Journal of Refrigeration, vol. 28, no. 3, pp. 442-450, 2004.

[15] J. G. Polihronov, A. G. Straatman, and C. T. DeGroot, "On the Thermodynamics of Angular Propulsion," in 10th International Conference on Heat Transfer, Fluid Mechanics and Thermodynamics. Orlando, FL: 10th International Conference on Heat Transfer, Fluid Mechanics and Thermodynamics, 2014.

[16] O. Tlili El May, I. Mokni, H. Mhiri, and P. Bournot, "CFD investigation of a vortex tube : Effect of the cold end orifice in the temperature separation mechanism," Science Academy Transactions on Renewable Energy Systems Engineering and Technology, vol. 1, no. 3, pp. 84-89, 2011.

[17] K. Masatsuka, I do like CFD, 2nd ed. Cradle, 2013, vol. 1.

[18] ANSYS Inc, "Workbench User's Guide," pp. 724-746, 2015.

[19] ANSYS Inc., “ANSYS CFX-Pre User's Guide,” pp. 724-746, 2011.

[20] T. Farouk and B. Farouk, "Large eddy simulations of the flow field and temperature separation in the Ranque-Hilsch vortex tube," International Journal of Heat and Mass Transfer, vol. 50, pp. 4724-4735, 2007. 